\title{
Performance evaluation of three laboratory diagnostic methods for intestinal parasitic infections at rural Bahir Dar, Northwest Ethiopia: A cross-sectional study
}

\author{
Birtukan Bayayibign¹, Mulat Yimer²*, Woynshet Gelaye² \\ 'Felege Hiwot Referral Specialized Hospital, Medical Laboratory Service, Bahir Dar, Ethiopia, \\ ${ }^{2}$ Department of Medical Laboratory Sciences, School of Health Sciences, College of Medicine and Health Science, Bahir \\ Dar University, Ethiopia
}

\begin{abstract}
Introduction: Even if the prevalence of intestinal parasites is high in Ethiopia, we still use only direct wet mount method for laboratory diagnosis of intestinal parasitic infections, having low sensitivity, and this significantly increases false-negative results. Therefore, performance evaluation of three laboratory diagnostic methods is mandatory.
\end{abstract}

Methods: Single stool sample was collected from March 2018 to June 2018, among 211 school children, and processed using wet mount, modified Baermann (MB), and Ritchie's methods. The sensitivity and negative predictive values (NPVs) at 95\% confidence interval and Kappa values were calculated in terms of the gold standard method (the combined result of altogether).

Results: The overall prevalence of intestinal parasites was $60.2 \%$. The sensitivity and NPVs of wet mount, MB, and Ritchie's methods against the "Gold standard" test were $49.6 \%$ and $56.8 \%, 80.3 \%$ and $77.1 \%$, and $67.7 \%$ and $68.8 \%$, respectively.

Conclusions: MB showed the best, and wet mount showed least performances for the laboratory diagnosis of intestinal parasitic infections.

Keywords: Wet mount; modified Baermann; Ritchie's method; Rural Bahir Dar; ethiopia

\section{INTRODUCTION}

Intestinal parasitic infection is a condition in which the gastrointestinal tract of human is infected with

\footnotetext{
*Corresponding author: Mulat Yimer, Department of Medical Laboratory Sciences, School of Health Sciences, College of Medicine and Health Science, Bahir Dar University, Ethiopia. Tel.: +251943390361. Fax: 2510582205932.

E-mail: yimermulat37@gmail.com
}

Submitted: 03 March 2019 / Accepted: 29 March 2019

DOI: https://doi.org/10.17532/jhsci.2019.170 mated that about 3.5 billion people in the world are infected with intestinal parasites (2). They are more prevalent among school children as compared to the general population $(3,4)$. About $12 \%$ of the global disease burdens are observed among school children with age ranges from 5 to 14 years (4).

The most prevalent intestinal parasites that cause infection in the human gastrointestinal tract are Giardia lamblia, Entamoeba histolytica, and soil-transmitted helminths (STHs) Ascaris 
lumbricoides, Trichuris trichiura Hookworm species (Hook worm sp.), Strongyloides stercoralis, Taenia saginata, and Hymenolepis sp. $(1,5)$.

In Ethiopia, intestinal parasitic infection is the second most predominant cause of outpatient morbidity $(2,6)$. The most prevalent intestinal helminths are $A$. lumbricoides, followed by $T$. trichiura and Hookworm sp. $(7,8)$. Negussu et al. (9) reported that, in Ethiopia, the number of people living with STHs in endemic areas is estimated at 79 million, comprising 9.1 million pre-school aged, 25.3 million school-aged, and 44.6 million adults. Despite this prevalence, wet mount method has been still used as a diagnostic method at all levels of health facilities in Ethiopia. Thus, evaluating additional laboratory diagnostic methods for intestinal parasitic infections that has not been used before in the country is mandatory as discussed in subsequent paragraphs below.

Wet mount method is the most commonly and routinely used method for the diagnosis of intestinal parasitic infections in Ethiopia due to its feasibility as compared to other techniques $(10,11)$. However, it has limitations such as lack of sensitivity as it used a small amount of stool sample (11).

Modified Baermanns (MBs) method has a greater sensitivity to detect most intestinal parasites including larva of $S$. stercoralis (12). It can be used routinely in developing countries such as Ethiopia because it requires less costly materials, used in the absence of a centrifuge, and not time-consuming $(13,14)$.

Finally, Ritchie's method is the sedimentation concentration in diagnosis of a wide range of intestinal parasites. This method is used for detecting intestinal nematode eggs that are not detected by wet mount method (15). This method is also better to concentrate intestinal helminthic eggs and intestinal protozoan cyst in fecal samples present in small numbers $(2,16)$.

Lack of laboratory diagnostic methods having a better sensitivity and specificity for intestinal parasitic infections impairs appropriate patient management and accurate epidemiological data and thus limits the disease control measure. Hence, evaluating the performances of laboratory diagnostic methods is essential for improving the diagnostic performances of these methods.

\section{METHODS}

\section{Study area and design}

A school-based cross-sectional study was conducted on school children at Meshenti and Gedro elementary schools in rural Bahir Dar, from March 2018 to June 2018. Bahir Dar is a capital city of Amhara Region lying at an altitude of $1900 \mathrm{~m}$ above sea level, $1419 \mathrm{~mm}$ annual rainfall, and average annual temperature of $19.6^{\circ} \mathrm{C}$. Based on the (17) census conducted by the Central Statistical Agency of Ethiopia, Bahir Dar special zone has a total population of 221,991 , of whom 108,456 are men and 113,535 women and $81.16 \%$ are urban inhabitants.

\section{Sample size determination and sampling technique}

We used Buderer's formula to evaluate different diagnostic methods. Since there is no previous study done in the study area, $50 \%$ prevalence, $95 \%$ confidence interval (CI), 5\% marginal error, and $10 \%$ non-response rate, a total of 211 students were selected.

Finally, the sample size was proportionally allocated for each class and grade, taking the total number of students in each category into consideration. Class roster was used as a sampling frame, and a systematic random technique was employed to select study participants.

\section{Stool sample collection and processing}

All study participants were informed about the purpose of the study. About 20-22 g single stool sample was collected from each study participants using clean stool cup and processed by wet mount, $\mathrm{MB}$, and Ritchie's methods. Each method was evaluated against the gold standard (the combined results of the three methods altogether based on Bayesian rule) (18).

\section{Laboratory procedures of the three diagnostic methods}

Wet mount method

In the wet mount, fresh stool samples ( $2 \mathrm{mg}$ of stool) were put on a slide with wooden applicator, emulsified with a drop of physiological saline $(0.85 \%)$ for 
diarrheic and semi-solid samples. For formed stools, iodine was used. Then, covered with cover slide and examined under microscope using first $\times 10$ objectives and then $\times 40$ objectives.

\section{$M B$ method}

The test was performed by $2-5 \mathrm{~g}$ of fresh stool sample homogenized in $10 \mathrm{ml}$ of saline solution and filtered through surgical gauze into a $50 \mathrm{ml}$ plastic tube, which is then filled with more saline solution, plugged, and shaken vigorously. Then, the tube is left to stand for $45 \mathrm{~min}$, after which the supernatant is removed and a sample is taken from the bottom and put on a slide for microscopy (19).

\section{Ritchie's method}

For this, $0.5 \mathrm{~g}$ fresh stool sample was added in the sample collecting tube containing $2.5 \mathrm{ml}$ of formalin and $1 \mathrm{ml}$ of ethyl acetate and the sample was well mixed and then centrifuged. Finally, the supernatant was discarded, the sediment mixed and put on the microscope slide for examination (20).

\section{Quality control}

To maintain the reliability of the study findings, $15 \%$ were randomly selected and reexamined at the end by experienced laboratory technologist who was blind for the first examination result.

\section{Data analysis}

Data were analyzed using Statistical Package for the Social Sciences statistical software version 20. Since there is no gold standard method to detect intestinal parasites, the combined results of all methods altogether can be used. The sensitivity, specificity, and negative predictive values (NPVs) at 95\% CI and Kappa values of each technique were calculated against the gold standard.

\section{Ethical considerations}

Ethical clearance was obtained from Bahir Dar University College of Medicine and Health Science ethical review committee before start the study. A supportive letter was obtained from Amhara Regional Health Bureau. A written informed consent was also obtained from every study participant, parent, or guardian. Those study participants who were positive for intestinal parasites were referred to the nearby health centers for treatment.

\section{RESULTS}

A total of 211 study participants were participated in the study and $60.2 \%$ were positive by the gold standard method. The prevalence of intestinal parasites using 3wet mount, $\mathrm{MB}$, and Ritchie's methods was $29.9 \%, 48.3 \%$, and $40.8 \%$, respectively (Table 1).

The sensitivity, specificity, and NPVs of wet mount method for the diagnosis of intestinal parasitic infections were $49.6 \%, 100 \%$, and $56.8 \%$, respectively. Agreements of this method with the gold standard for the diagnosis of intestinal parasitic infections were moderate $(\mathrm{k}=0.439)$ (Table 2$)$.

The sensitivity, specificity, and NPVs of MB method were $80.3 \%, 100 \%$, and $77.1 \%$, respectively. Agreements of the test with the gold standard for the diagnosis of intestinal parasitic infections were very good $(\mathrm{k}=0.77)$ (Table 3$)$. The sensitivity, specificity, and NPV of Ritchie's method were $67.72 \%$, $100 \%, 67.2 \%$, respectively. Finally, agreements of the test with the gold standard for the diagnosis of intestinal parasitic infections were very good $(\mathrm{k}=0.63)($ Table 4).

\section{DISCUSSION}

In this study, the overall prevalence was $60.2 \%$. This was lower than a study in Northwest Ethiopia,

TABLE 1. The performance of diagnostic methods for intestinal parasitic infections

\begin{tabular}{lcccc}
\hline Results & & & & \\
\hline Methods & Number examined & Positive $n(\%)$ & No ova/parasite $n(\%)$ & Total $n(\%)$ \\
\hline Wet mount & 211 & $63(29.9)$ & $148(70.1)$ & $211(100)$ \\
MB & 211 & $102(48.3)$ & $109(51.7)$ & $211(100)$ \\
Ritchie's & 211 & $86(40.8)$ & $125(59.2)$ & $211(100)$ \\
All methods & 211 & $127(60.2)$ & $84(39.8)$ & $211(100)$ \\
\hline
\end{tabular}

MB: Modified Baermann 
TABLE 2. The performance of wet mount method against the gold standard for the diagnosis of intestinal parasitic infections (\%)

Gold standard method

\begin{tabular}{lccccccc}
\hline Wet mount & Positive & Negative & Total & Sensitivity (95\% Cl) & Specificity (95 \% Cl) & NPV (95\% Cl) & Kappa \\
\hline Positive & $63(49.6)$ & $0(0)$ & $63(29.9)$ & $49.6[58.8-75.7]$ & $100[95.7-100]$ & $56.7[61.4-72.5]$ & 0.63 \\
Negative & $64(50.4)$ & $84(100)$ & $148(70.1)$ & & & & \\
Total & $127(100)$ & $84(100)$ & $211(100 \%)$ & & & & \\
\hline
\end{tabular}

$\mathrm{Cl}$ : Confidence interval

TABLE 3. The performance of MB against the gold standard method for the diagnosis of intestinal parasitic infections

\begin{tabular}{lccccccc}
\hline \multicolumn{6}{l}{ Gold standard method } & \multicolumn{7}{l}{} \\
\hline MB & Positive (\%) & Negative (\%) & Total (\%) & Sensitivity (95\% Cl) & Specificity (95\% Cl) & NPV (95\% Cl) & Kappa \\
\hline Positive & $102(80.3)$ & $0(0)$ & $102(48.3)$ & & & & \\
Negative & $25(19.7)$ & $84(100)$ & $109(51.7)$ & $80.3[72.3-86.8]$ & $100[95.7-100]$ & $53.5[70.3-82.7]$ & 0.77 \\
Total & $127(100)$ & $84(100)$ & $211(100)$ & & & \\
\hline
\end{tabular}

$\mathrm{Cl}$ : Confidence interval, MB: Modified Baermann

TABLE 4. The performance of Ritchie's method against the Gold standard for the diagnosis of intestinal parasitic infections

\begin{tabular}{lccccccc}
\hline \multicolumn{6}{l}{ Gold standard method } & \multicolumn{5}{l}{$l$} \\
\hline Ritchie's & Positive & Negative & Total & Sensitivity $(95 \% \mathrm{Cl})$ & Specificity $(95 \% \mathrm{Cl})$ & NPV $(95 \% \mathrm{Cl})$ & Kappa \\
\hline Positive & $86(67.7)$ & $0(0)$ & $86(67.7)$ & & & & \\
Negative & $41(32.3)$ & $84(100)$ & $41(32.3)$ & $67.72[58.8-75.7]$ & $100[95.7-100]$ & $67.2[61.4-72.5]$ & 0.63 \\
Total & $127(100)$ & $84(100)$ & $211(100)$ & & & & \\
\hline
\end{tabular}

$\mathrm{Cl}$ : Confidence interval

79.8\% (21) and East Gojjam Zone 83.4\% (22) and higher than 35.44\% in Benishangul-Gumuz (23) and in Bahir Dar, Northwest, 59.8\% (4). This difference might be due to the geographical difference, or it might be associated with a difference in parasitological methods.

In this study, wet mount method confirmed 29.9\%, modified Baermann confirmed 48.3\%, and Ritchie's method confirmed $40.8 \%$. This result agrees with the previous studies $(13,14)$.

Wet mount method exhibited very low sensitivity for the detection of other intestinal helminths. This was in agreement with a study done in Bahir Dar (4). A small amount of fecal material used in this technique might be the reason for lower detection capacity of the method. Wet mount method exhibited the lowest sensitivity of $49.6 \%$ and NPVs of $56.76 \%$ as compared to MB, with a sensitivity of $80.3 \%$ and NPVs of $77.1 \%$, and Ritchie's method with sensitivity $67.7 \%$ and NPVs of $68.8 \%$ for the detection intestinal parasitic infections. This suggested that the use of wet mount method for the diagnosis of intestinal parasitic infections is insufficient and the use of another diagnosing method is mandatory to decrease misdiagnosis.

In the current study, $48.3 \%$ of the intestinal parasitic infections were detected by the MB method with a sensitivity of $80.3 \%$ and NPVs of $77.1 \%$. This method is promising to use it as a routine laboratory diagnostic method for intestinal parasitic infections. Moreover, MB requires less costly materials even we can use in the absence of centrifuge. However, lack of previous similar studies made difficulty in making rigorous discussion on this finding. In the present study, Ritchie's method detected $23.7 \%$ of Hookworm sp. followed by $A$. lumbricoides $5.7 \%$. This is in agreement with other studies done previously (24). However, this study disagrees with a study done in Rio de Janeiro, Brazil. This high value may be due to the use of greater size of the coverslips measuring $24 \mathrm{~mm} \times 32 \mathrm{~mm}$ to increase the spread of fecal material than those used in routine laboratory analysis $(22 \mathrm{~mm} \times 22 \mathrm{~mm})(25)$. 
Based on parasite recovery, our results confirmed that Ritchie's method (40.8\%) is higher than the direct wet mount method (29.9\%). This result was in agreement with other studies done previously $(2,26)$. Furthermore, the sensitivity and NPVs of Ritchie's method were $67.7 \%$ and $67.2 \%$ which were higher than wet mount method $49.6 \%$ and $56.8 \%$.

\section{CONCLUSIONS}

In this study, MB showed the best performance as compared to wet mount and Ritchie's methods, and Ritchie's method showed better performance as compared to wet mount method. Therefore, it is preferable to use $\mathrm{MB}$, in complement with Ritchie's and wet mount methods as a routine laboratory diagnosis of intestinal parasitic infections.

\section{Limitation of the study}

The limitation of this study was that all the combined results of the three methods are highly influenced by parasite prevalence. Thus, the same method will have different values in different areas of prevalence.

\section{ACKNOWLEDGMENTS}

We would like to express great thanks to participants from Gedro and Meshenti elementary schools' children. Moreover, we would also like to express great thanks to the directors of two schools and those who were involved in data collection process.

\section{COMPETING OF INTEREST}

We authors declare that we have no conflicts of interest.

\section{REFERENCES}

1. Haque R. Human intestinal parasites. I Health Popul Nutr 2007;25(4):387-91.

2. Endris M, Tekeste Z, Lemma W, Kassu A. Comparison of the kato-katz, wet mount, and formol-ether concentration diagnostic techniques for intestinal helminth infections in Ethiopia. ISRN Parasitol 2012;5(10):402-39.

3. Andualem M. Parasitic infection and associated factors among the primary school children in Motta town, Western Amhara, Ethiopia. Am J Public Health Res 2014;2(6):248-54. https://doi.org/10.12691/ajphr-2-6-6.

4. Hailegebriel T. Prevalence of intestinal parasitic infections and associated risk factors among students at Dona Berber primary school, Bahir Dar,
Ethiopia. BMC Infect Dis 2017;17:362.

https://doi.org/10.1186/s12879-017-2466-x.

5. Daryani A, Hosseini-Teshnizi S, Hosseini SA, Ahmadpour E, Sarvi S, Amouei $A$, et al. Intestinal parasitic infections in Iranian preschool and school children: A systematic review and meta-analysis. Acta Trop 2017;169:69-83.

https://doi.org/10.1016/j.actatropica.2017.01.019.

6. Yimer M, Hailu T, Mulu W, Abera B. Evaluation performance of diagnostic methods of intestinal parasitosis in school age children in Ethiopia. BMC Res Notes 2015;8:820.

https://doi.org/10.1186/s13104-015-1822-4.

7. Belyhun $Y$, Medhin G, Amberbir A, Erko B, Hanlon C, Alem A, et al. Prevalence and risk factors for soil-transmitted helminth infection in mothers and their infants in Butajira, Ethiopia: A population based study. BMC Public Health 2010;10:21.

https://doi.org/10.1186/1471-2458-10-21.

8. Derso A, Nibret E, Munshea A. Prevalence of intestinal parasitic infections and associated risk factors among pregnant women attending antenatal care center at felege hiwot referral hospital, Northwest Ethiopia. BMC Infect Dis 2016;16:530.

https://doi.org/10.1186/s12879-016-1859-6.

9. Negussu N, Mengistu B, Kebede B, Deribe K, Ejigu E, Tadesse G, et al. Ethiopia schistosomiasis and soil-transmitted helminthes control programme: Progress and prospects. Ethiop Med J 2017;55(1):75-80.

10. Tankeshwar A. Direct saline or iodine Wet Mount for diagnosis of intestinal parasites. Clin Microbiol Infect 2015;5(3):1-5.

11. Hailu T, Abera B. Performance evaluation of direct saline stool microscopy, formol ether concentration and kato katz diagnostic methods for intestinal parasitosis in the absence of gold standard methods. Trop Doct 2015;45(3):178-82.

https://doi.org/10.1177/0049475515581127.

12. Siddiqui AA, Berk SL. Diagnosis of Strongyloides stercoralis infection. Clin Infect Dis 2001;33:1040-7.

https://doi.org/10.1086/322707.

13. Gallardo MS, Cornejo M, Vasquez G, Errea R, Urquiaga J, Montoya D. High prevalence of intestinal parasites among soldiers in Peru: Another population at risk Peru. J Parasitol 2011;19(2):60-7.

14. Tello R, Terashima A, Marcos LA, Machicado J, Canales M, Gotuzzo E, et al. Highly effective and inexpensive parasitological technique for diagnosis of intestinal parasites in developing countries: Spontaneous sedimentation technique in tube. Int J Infect Dis 2012;16(6):e414-6.

https://doi.org/10.1016/j.ijid.2011.12.017.

15. Jorge D, Luis A, Tello R, Canales M, Terashima A, Gotuzzo E. Diagnosis of soil-transmitted helminthiasis in an amazon community of Peru using multiple diagnostic techniques. Trop Med Hyg 2012;106:333-9.

https://doi.org/10.1016/j.trstmh.2012.03.004.

16. Requena-Méndez A, Chiodini P, Bisoffi Z, Buonfrate D, Gotuzzo E, Muñoz J, et al. The laboratory diagnosis and follow up of strongyloidiasis: A systematic review. PLoS Negl Trop Dis 2013;7(1):e2002.

17. Federal Democratic Republic of Ethiopia Population Census Commission. Summary and Statistical Report of the 2007 Population and Housing Census: Population Size by Age and Sex. Addis Ababa: Centreral Statistics Agency; 2008.

18. Dendukuri N, Rahme E, Bélisle P, Joseph L. Bayesian sample size determination for prevalence and diagnostic test studies in the absence of a gold standard test. Biometrics 2004;60(2):388-97.

https://doi.org/10.1111/j.0006-341X.2004.00183.x.

19. Buonfrate D, Formenti F, Perandin F, Bisoffi Z. Novel approaches to the diagnosis of Strongyloides stercoralis infection. Clin Microbiol Infect 2015;21(6):543-52. 
https://doi.org/10.1016/j.cmi.2015.04.001.

20. Anécimo RS, Tonani KA, Fregonesi BM, Mariano AP, Ferrassino MD, Trevilato TM, et al. Adaptation of Ritchie's method for parasites diagnosing with minimization of chemical products. Interdiscip Perspect Infect Dis 2012;2012:409757.

https://doi.org/10.1155/2012/409757.

21. Ayalew A, Debebe T, Worku A. Prevalence and risk factors of intestinal parasites among Delgi school children, North Gondar. J Parasitol Vector Biol 2011;3(5):75-81.

22. Workneh T, Esmael A, Ayichiluhm M. Prevalence of intestinal parasitic infections and associated factors among Debre Elias primary schools children, East Gojjam zone, Amhara region, North West Ethiopia. J Bacteriol Parasitol 2014;5:181.

https://doi.org/10.4172/2155-9597.1000181.

23. Gebretsadik G. Prevalence of intestinal parasites and associated risk factors among schoolchildren of Homesha district in Benishangul-Gumuz regional state, Western Ethiopia. J Fam Med Health Care 2016;2(4):57-64. https://doi.org/10.11648/j.jfmhc.20160204.16.

24. Amin H, Ali S. Evaluation of different techniques of stool examination for intestinal parasitic infections in Sulaimani City-Iraq. Microbiol Appl Sci 2015;4(5):991-6.

25. Barbosa Ada S, Bastos OM, Uchôa CM, Pissinatti A, Bastos AC, Souza IV et al. Comparison of five parasitological techniques for laboratory diagnosis of Balantidium coli cysts. Rev Bras Parasitol Vet 2016;25(3):286-92. https://doi.org/10.1590/S1984-29612016044.

26. Moges F, Belyhun Y, Tiruneh M, Kebede Y, Mulu A, Kassu A. Comparison of formol-acetone concentration method with that of the direct iodine preparation and formol-ether concentration methods for examination of stool parasites. Ethiop J Health Dev 2010;24:148-51.

https://doi.org/10.4314/ejhd.v24i2.62965. 